Supporting Information

\title{
Atomic Layer Deposition of Ru for Replacing Cu-interconnects
}

Yohei Kotsugi ${ }^{\mathrm{a}}$, b, Seung-Min Hanc, Youn-Hye Kimc, Taehoon Cheon ${ }^{\mathrm{c}}$, Dip K. Nandic ${ }^{\mathrm{c}}$, Rahul Ramesh ${ }^{\mathrm{c}}$, Neung Kyung $\mathrm{Yu}^{\mathrm{d}}$, Kirak Son ${ }^{\mathrm{e}}$, Tomohiro Tsugawa ${ }^{\mathrm{a}}$, Shigeyuki Ohtake ${ }^{\mathrm{a}}$, Ryosuke Harada $^{\mathrm{a}}$, Young-Bae Park ${ }^{\mathrm{e}}$, Bonggeun Shong ${ }^{\mathrm{d}}$, and Soo-Hyun Kim ${ }^{\mathrm{b}, \mathrm{c}}$ *

a Chemical Materials Development Department, TANAKA Precious Metals, 22, Wadai, Tsukuba, Ibaraki, 3004247, Japan

b Institute of Materials Technology, Yeungnam University, Yeungnam University, 280 Daehak-Ro, Gyeongsan, Gyeongbuk, 38541, Republic of Korea

Email: soohyun@ynu.ac.kr

${ }^{c}$ School of Materials Science and Engineering, Yeungnam University, Gyeongsan, Gyeongbuk, 38541, Republic of Korea

d Department of Chemical Engineering, Hongik University, Mapo-gu, Seoul, 04066, Republic of Korea

Email: bshong@hongik.ac.kr

e School of Materials Science and Engineering, Andong National University, Andong, Gyeongsangbuk, 36729, Republic of Korea 
Table S1. Summary of the FWHM of Ru(101) peaks and the grain size calculated from Scherrer formula.

\begin{tabular}{|c|c|c|}
\hline $\begin{array}{c}\text { Deposition } \\
\text { temperature }\left({ }^{\circ} \mathrm{C}\right)\end{array}$ & $\begin{array}{c}\mathrm{FWHM}\left({ }^{\circ}\right) \\
\text { of } \mathrm{Ru}(101) \text { peak }\end{array}$ & Grain size (nm) \\
\hline 180 & 0.583 & 19.0 \\
\hline 220 & 0.624 & 17.7 \\
\hline 260 & 0.554 & 20.0 \\
\hline 300 & 0.539 & 20.5 \\
\hline
\end{tabular}




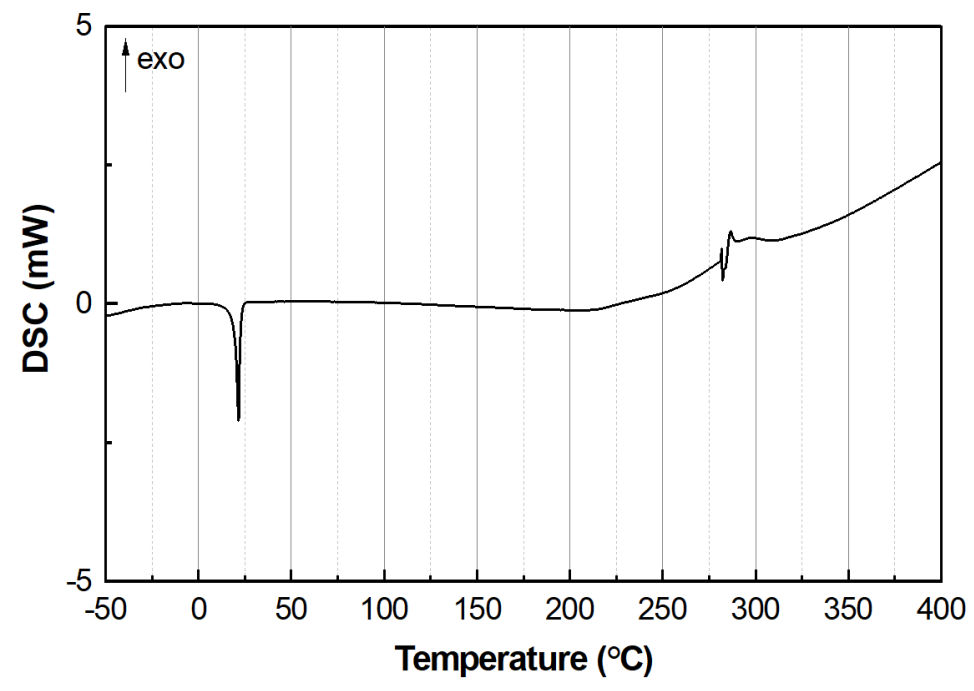

Figure S1. DSC curve of $\left[\mathrm{Ru}(\mathrm{TMM})(\mathrm{CO})_{3}\right]$ which was analyzed in the sealed aluminium pan perged by $\mathrm{N}_{2}$ gas. 

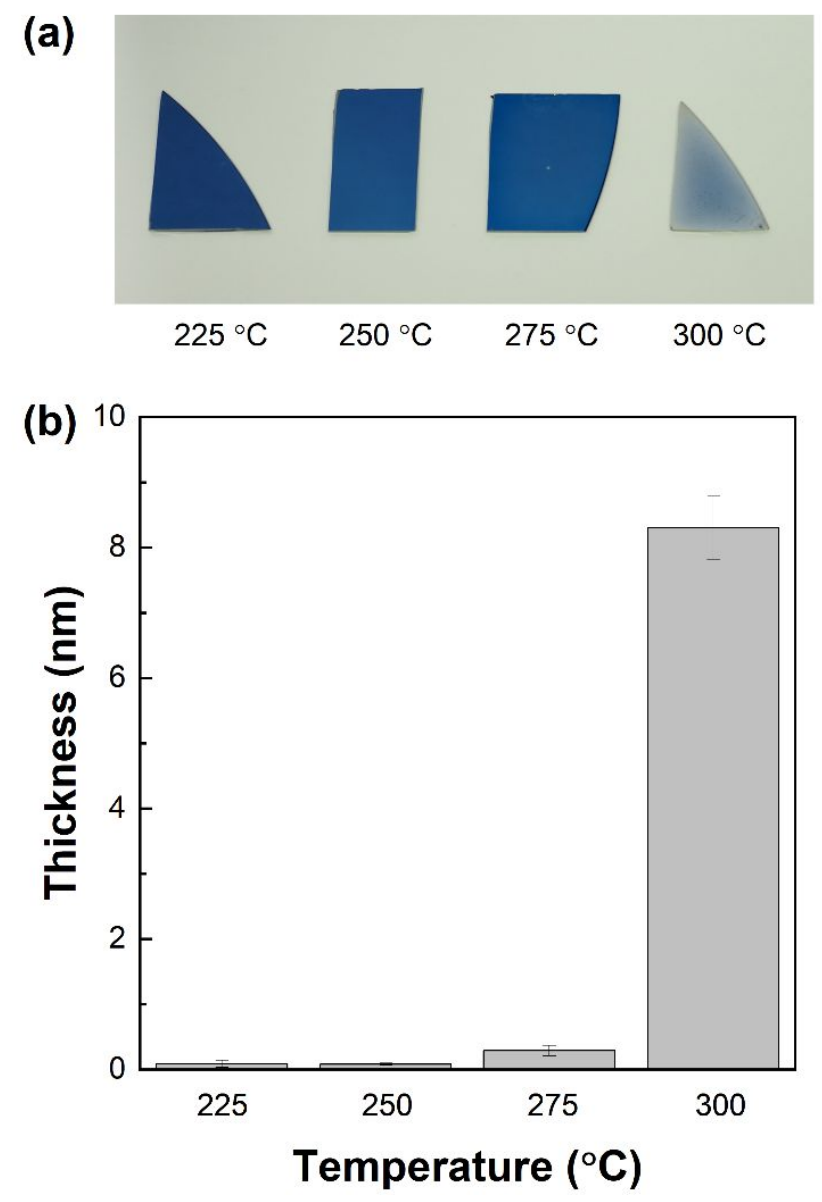

Figure S2. (a) Appearance and (b) thickness determined by XRF of films which were thermally decomposited on a thermally grown $\mathrm{SiO}_{2}$ substrate at $225,250,275$ and $300{ }^{\circ} \mathrm{C}$ without any reactant gases. 


\section{Average thickness: $35.9 \mathrm{~nm}$}

\section{$10.0 \mathrm{kV} 9.6 \mathrm{~mm} \times 100 \mathrm{k}$}

Figure S3. Thickness of the ALD-Ru films deposited on a thermally grown $\mathrm{SiO}_{2}$ substrate at $220{ }^{\circ} \mathrm{C}$ under the optimized pulsing conditions measured by cross-sectional view SEM. 

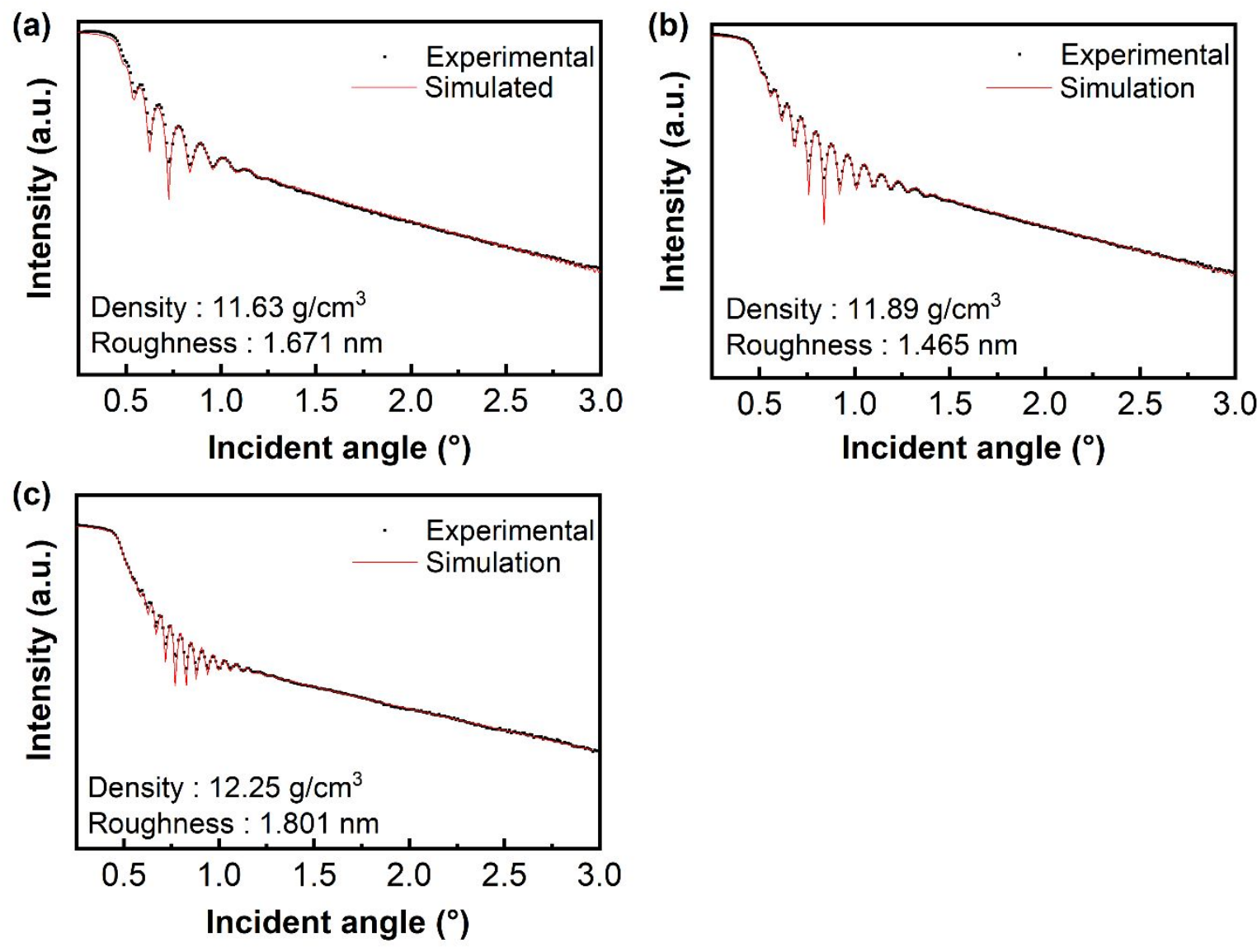

Figure S4. Density and roughness of the films deposited at (a) 180, (b) 220 and (c) $300{ }^{\circ} \mathrm{C}$ simulated by XRR. 


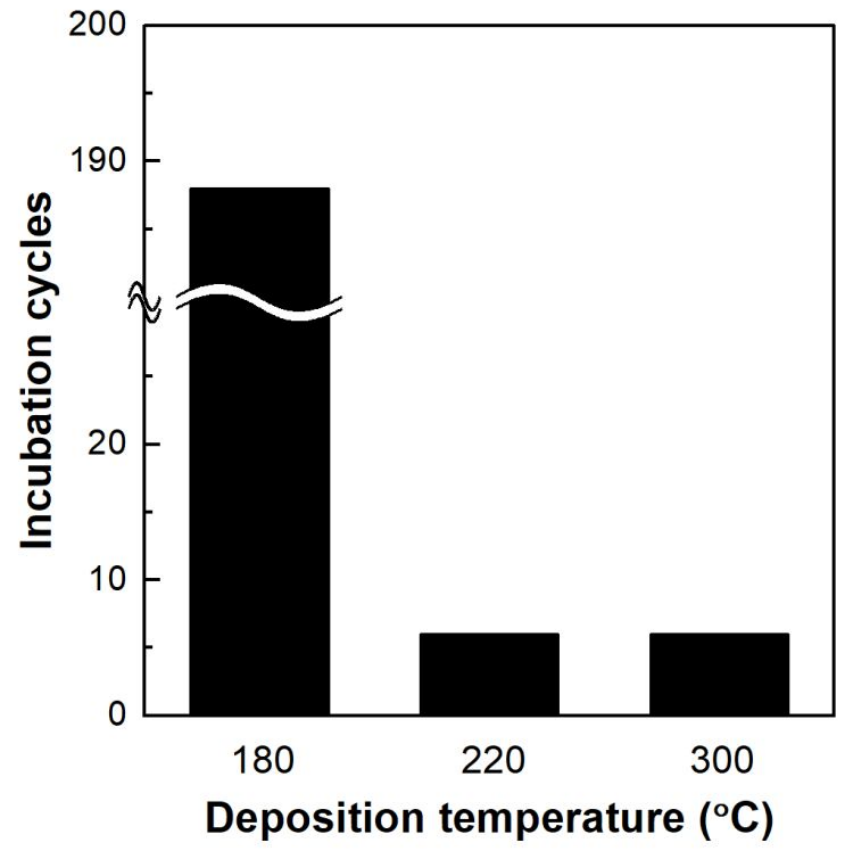

Fig. S5. The incubation cycles at each deposition temperatures of 180,220 and $300^{\circ}$ C. 


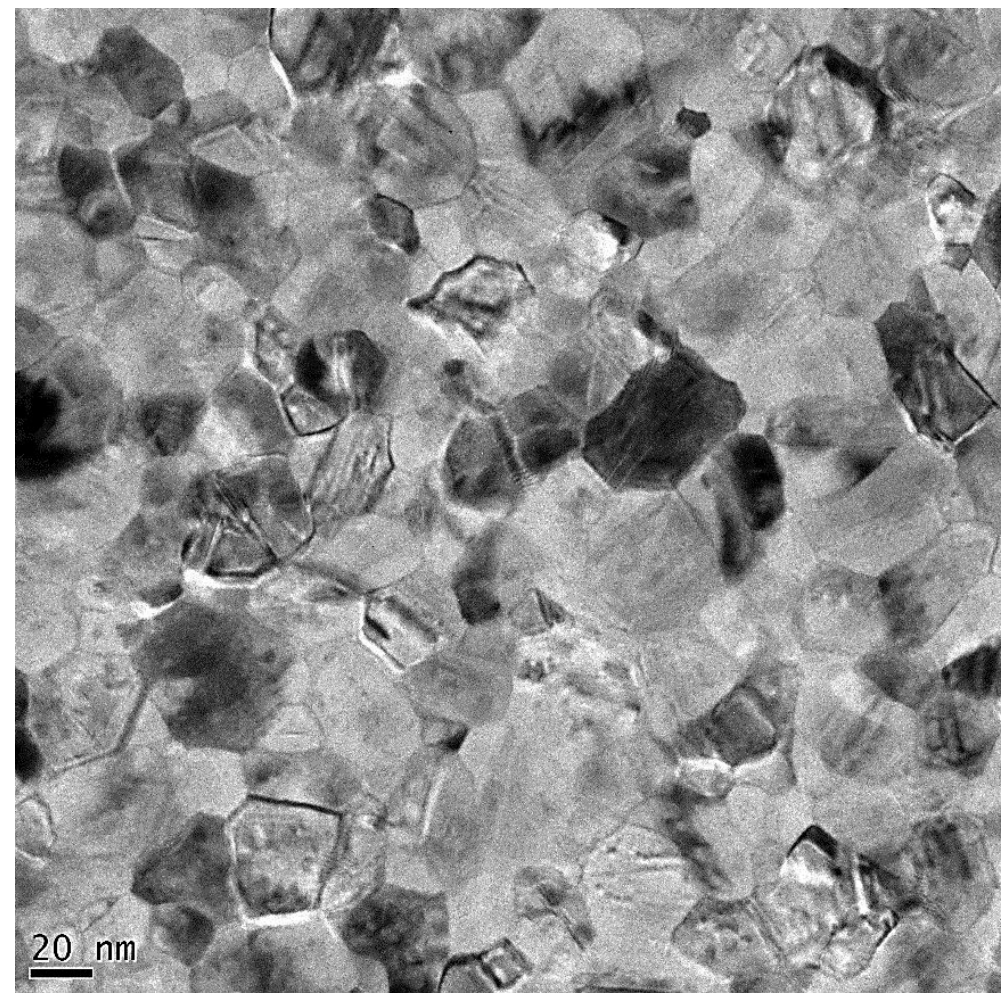

Figure S6. Plan-view bright-field TEM image of ALD-Ru film deposited on a TiN substrate under optimized pulsing conditions at $240{ }^{\circ} \mathrm{C}$. 


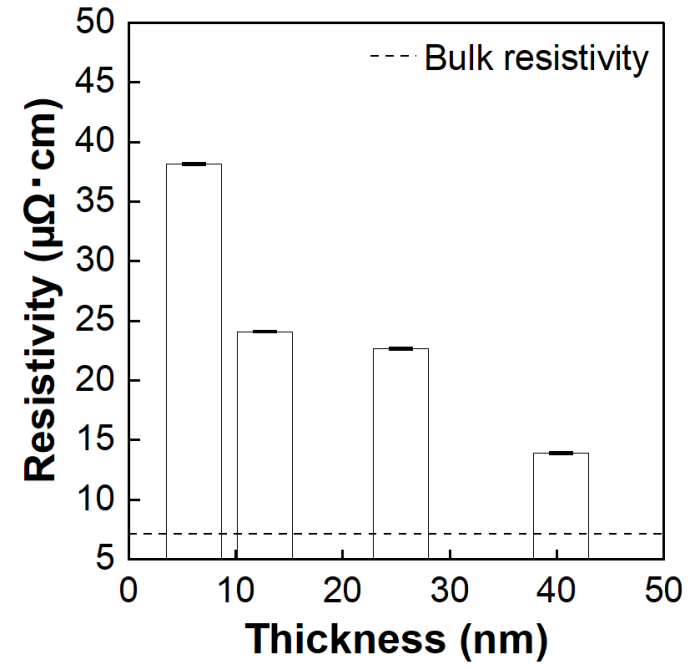

Figure S7. Resistivity of the ALD-Ru films deposited on a thermally-grown $\mathrm{SiO}_{2}$ substrate at $220{ }^{\circ} \mathrm{C}$ under optimized pulsing conditions as a function of the film thickness. 


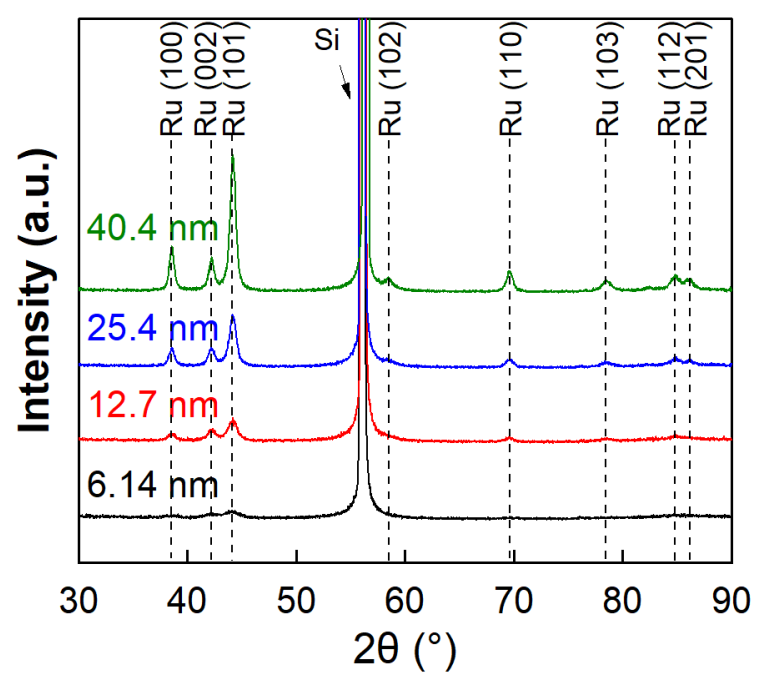

Figure S8. GIAXRD patterns of the ALD-Ru films deposited on a thermally-grown $\mathrm{SiO}_{2}$ substrate at $220^{\circ} \mathrm{C}$ under optimized pulsing conditions as a function of the film thickness. 


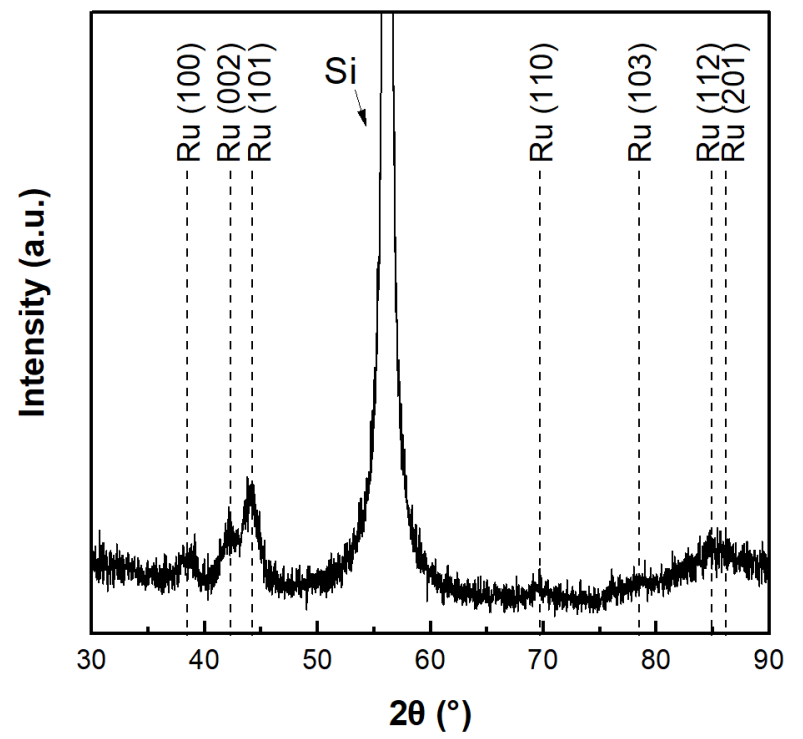

Figure S9. GIAXRD patterns of the $\sim 6 \mathrm{~nm}$ ALD-Ru film deposited on a thermally grown $\mathrm{SiO}_{2}$ substrate at $220{ }^{\circ} \mathrm{C}$ under the optimized pulsing conditions. 


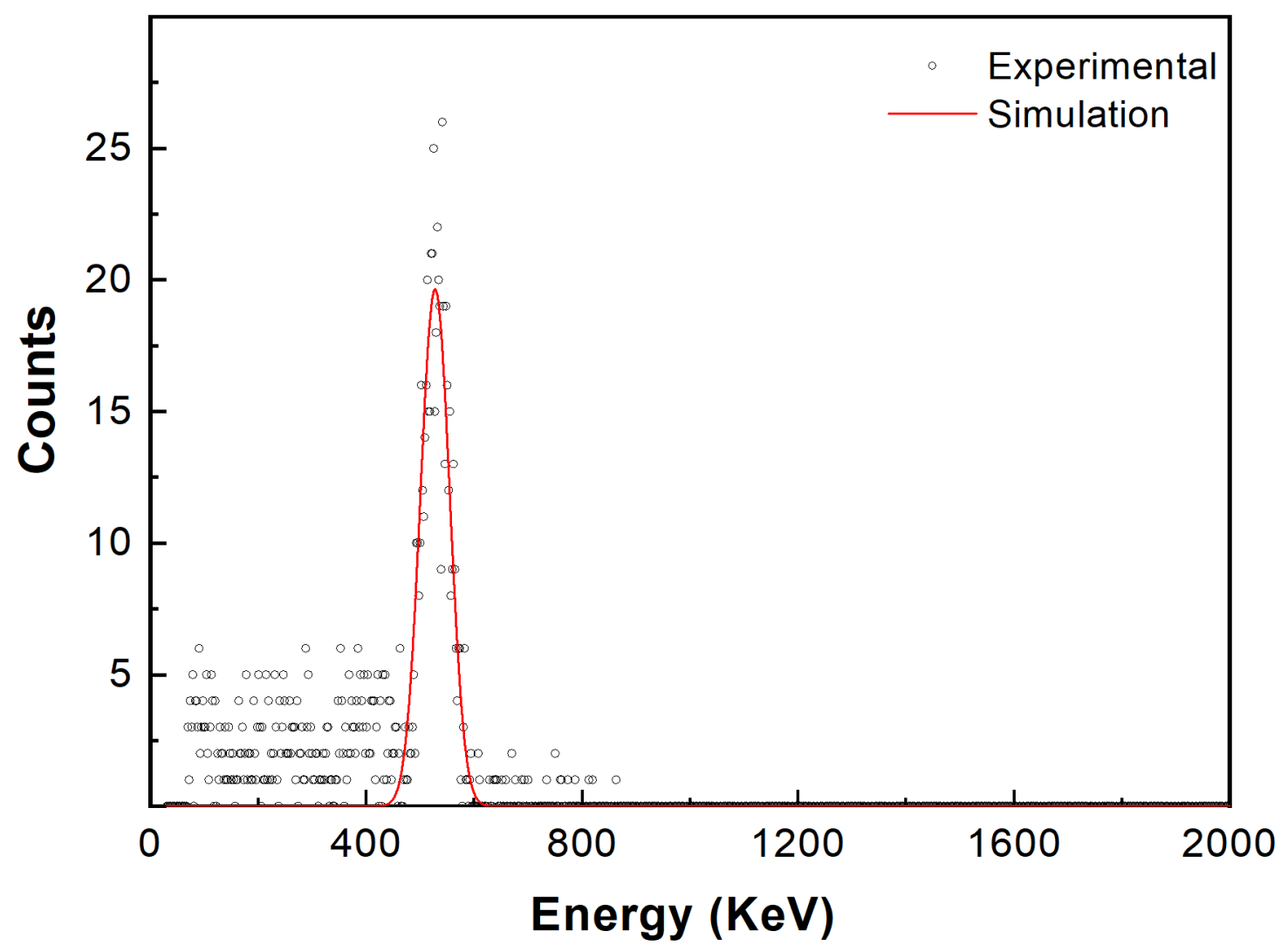

Figure S10. ERD of the ALD-Ru film deposited on a Si substrate under the optimized pulsing conditions at $220{ }^{\circ} \mathrm{C}$. 


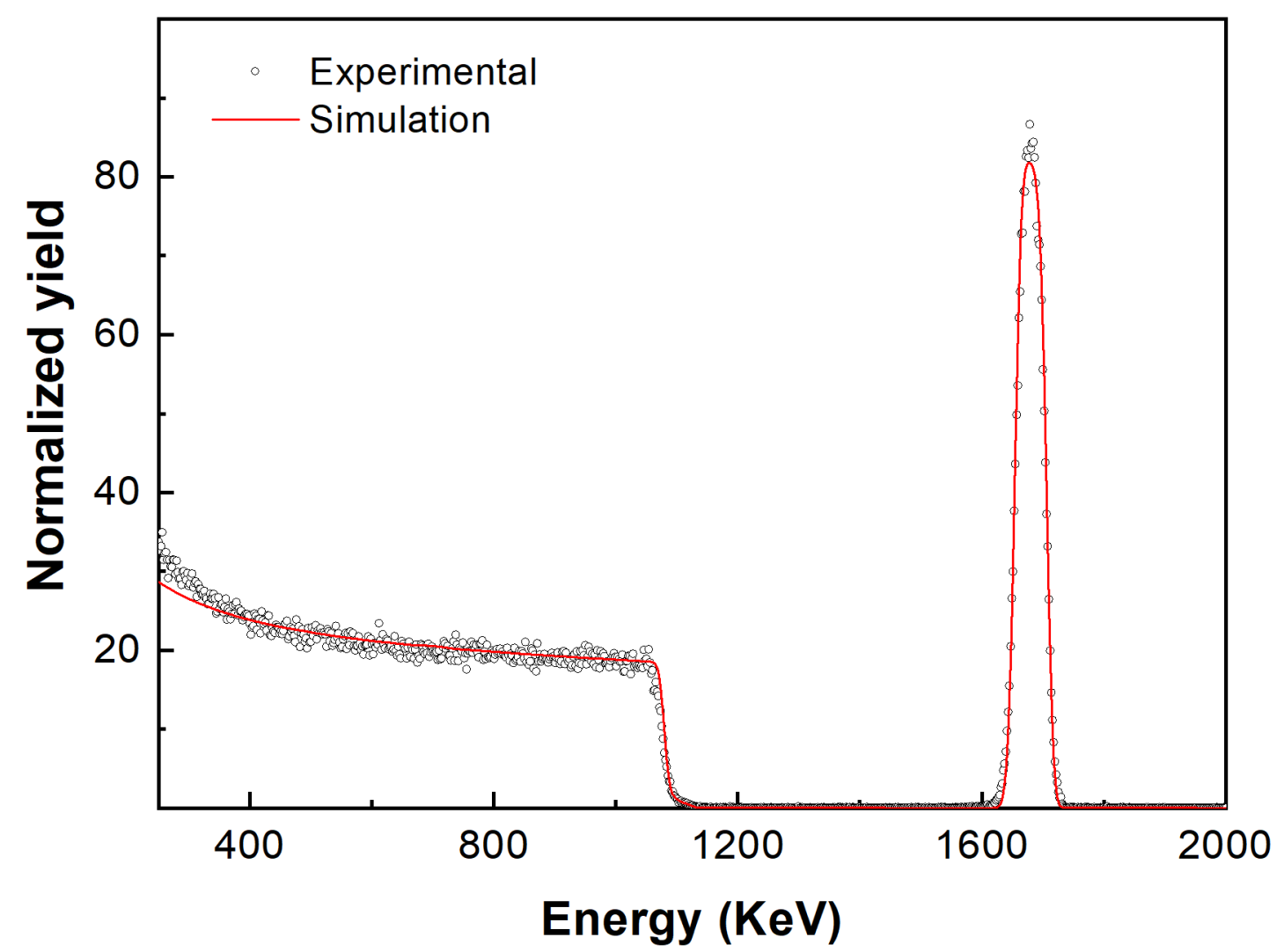

Figure S11. RBS and the simulation curve with ratio determined by ERD and TOF-ERD of the ALD-Ru film deposited on a Si substrate under the optimized pulsing conditions at $220{ }^{\circ} \mathrm{C}$ 


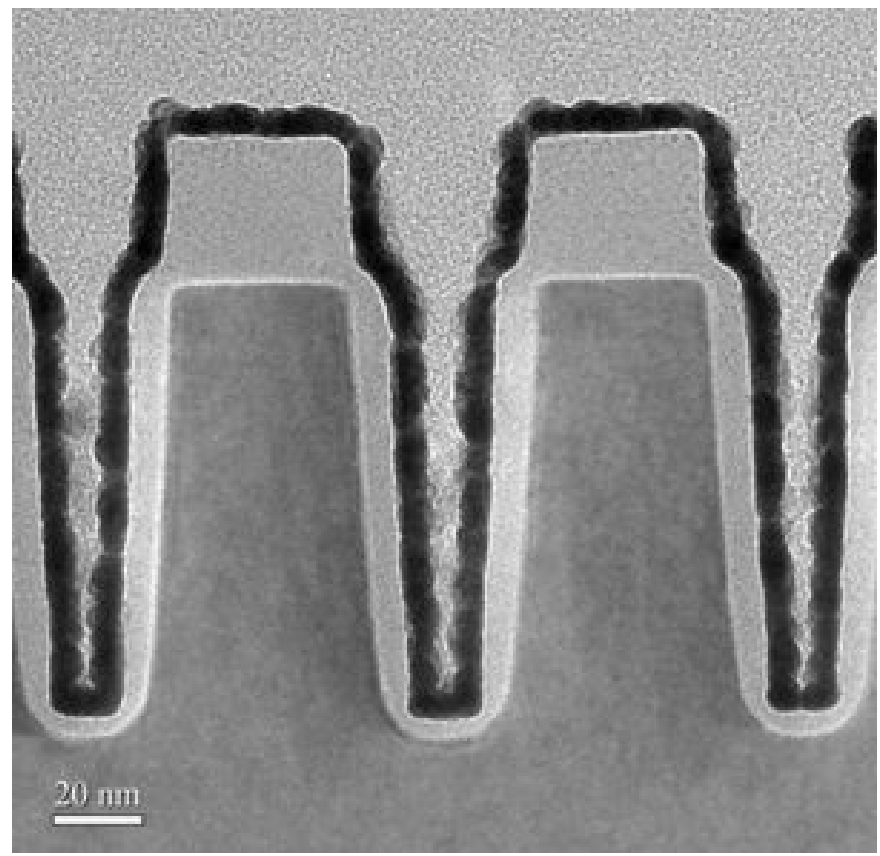

Figure S12. XTEM images with a high magnification of the ALD-Ru film deposited @ $220{ }^{\circ} \mathrm{C}$ on dual trench substrate to determine the step coverage. 


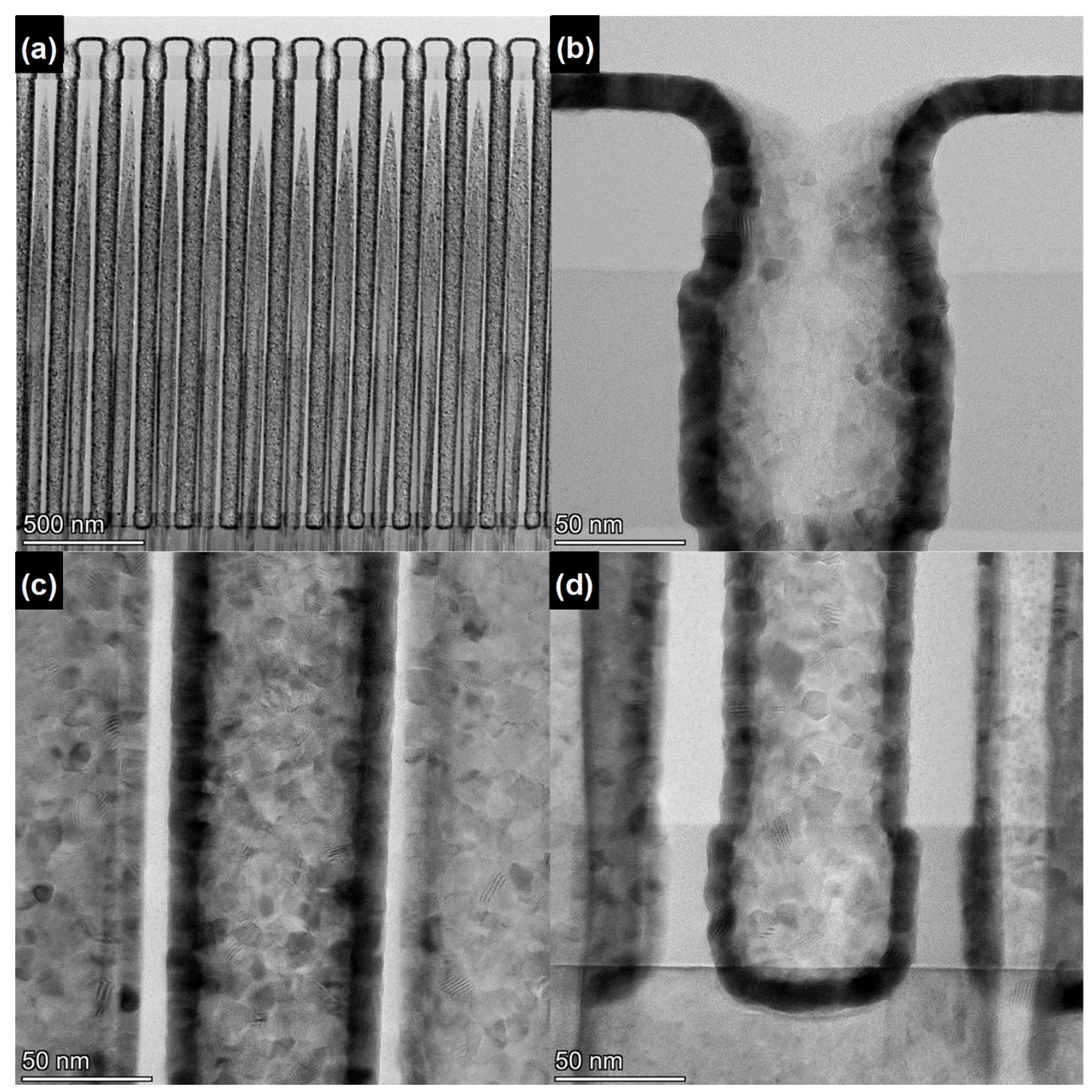

Figure S13. XTEM images of the ALD-Ru film deposited $@ 260{ }^{\circ} \mathrm{C}$ on the hole substrate to determine the step coverage; (a) overview with low magnication, (b) at the top, (c) the middle, and (d) bottom of the hole. 


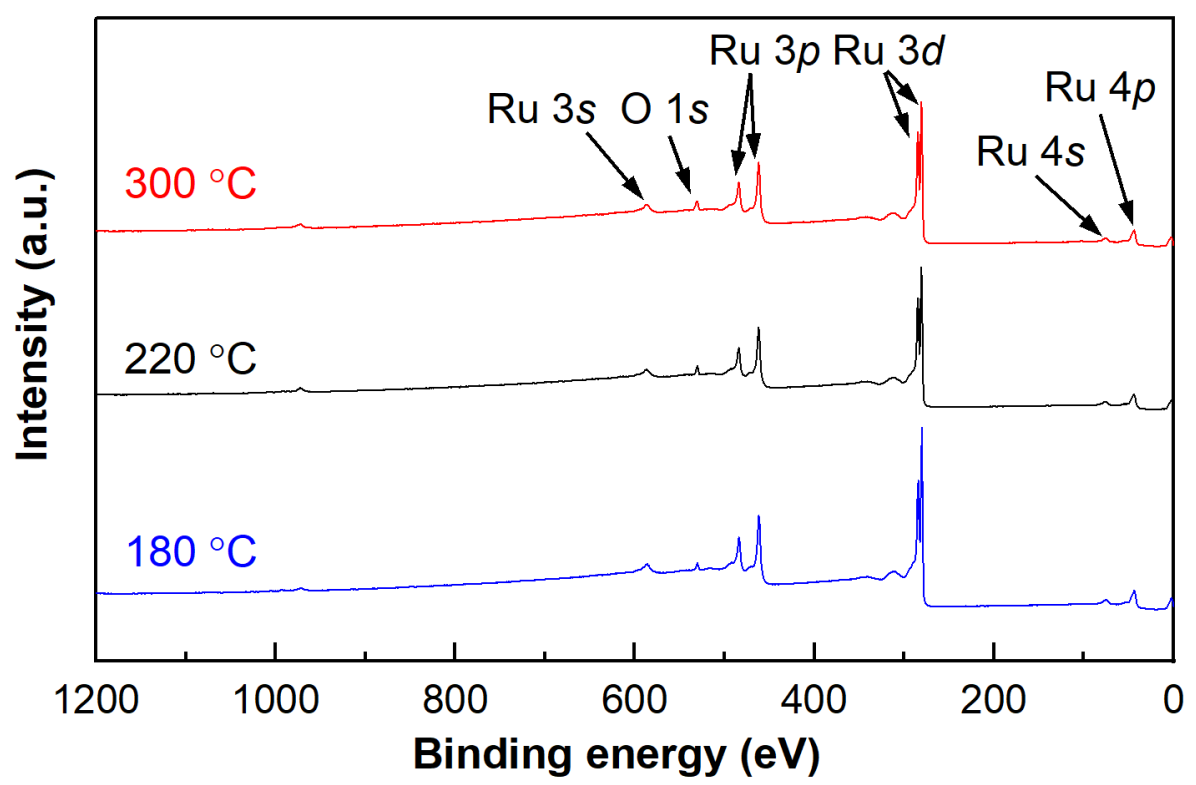

Figure S14. X-ray photoelectron survey spectra of Ru films deposited at 180,220 and $300{ }^{\circ} \mathrm{C}$. 

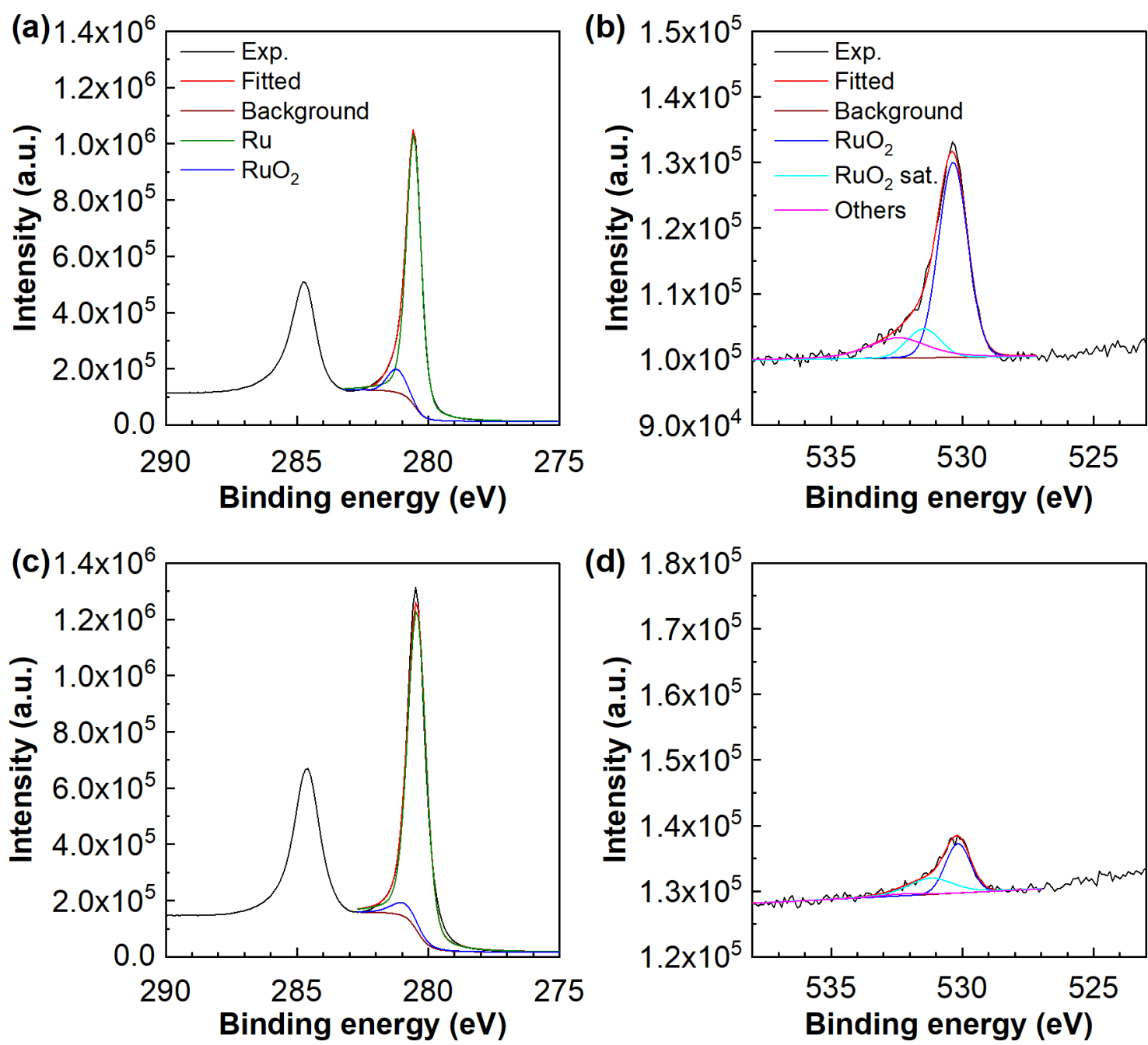

Figure S15. X-ray photoelectron spectra for Ru $3 d$ and $\mathrm{O} 1 s$ of Ru thin-films (a), (b) deposited at $220{ }^{\circ} \mathrm{C}$ and (c), (d) post annealed at $500{ }^{\circ} \mathrm{C}$. 